\title{
Evolution of the Io footprint brightness I: Far-UV observations
}

\author{
B. Bonfond ${ }^{\mathrm{a}, \mathrm{b}, *}$, S. Hess ${ }^{\mathrm{c}}$, J.-C. Gérard ${ }^{\mathrm{a}}$, D. Grodent ${ }^{\mathrm{a}}$, A. Radioti ${ }^{\mathrm{a}}$, V. Chantry ${ }^{\mathrm{d}}$, J. Saur ${ }^{\mathrm{e}}$, \\ S. Jacobsen ${ }^{\text {e,f }}$, J.T. Clarke ${ }^{g}$ \\ a Laboratoire de Physique Atmosphérique et Planétaire (LPAP), Université de Liège, Liège, Belgium \\ ${ }^{\mathrm{b}}$ Department of Space Studies, Southwest Research Institute, Boulder, Colorado, USA \\ ${ }^{\mathrm{c}}$ LATMOS, Université Versailles-St Quentin, IPSL/CNRS, Guyancourt, France \\ ${ }^{\mathrm{d}}$ Astrophysique et Traitement de l'Image (ATI), Université de Liège, Liège, Belgium \\ e Institut für Geophysik und Meteorologie, Universität zu Köln, Köln, Germany \\ ${ }^{\mathrm{f}}$ Research and Technology Centre Westcoast, Universität zu Kiel, Büsum, Germany \\ ${ }^{g}$ Center for Space Physics, Boston University, USA
}

\section{A R T I C L E I N F O}

\section{Article history:}

Received 28 November 2012

Received in revised form

29 May 2013

Accepted 31 May 2013

Available online 13 June 2013

Keywords:

Jupiter

Io

Aurora

Io footprint

Hubble space telescope

\begin{abstract}
A B S T R A C T
The Io footprint (IFP) is a set of auroral spots and an extended tail resulting from the strong interaction between Io and the Jovian magnetosphere. For the first time, we present measurements of the brightness and precipitated power for each individual spot, using the image database gathered from 1997 to 2009 with the Hubble Space Telescope in the Far-UV domain. We show that the relative brightness of the spots varies with the System III longitude of Io. Moreover, our novel measurement method based on 3D simulations of the auroral features allows to derive the precipitated energy fluxes from images on which the emission region is observed at a slant angle. Peak values as high as $2 \mathrm{~W} / \mathrm{m}^{2}$ are observed for the main spot, probably triggering a localized and sudden heating of the atmosphere. Additionally, strong brightness differences are observed from one hemisphere to another. This result indicates that the location of Io in the plasma torus is not the only parameter to control the brightness, but that the magnetic field asymmetries also play a key role. Finally, we present new data confirming that significant variations of the spots' brightness on timescales of 2-4 min are ubiquitous, which suggests a relationship with intermittent double layers close to Jovian surface.
\end{abstract}

\section{Introduction}

Io, Jupiter's volcanic moon, releases approximately one ton of $\mathrm{SO}_{2}$ every second. Half of this material, once ionized, temporarily remains in the Jovian magnetosphere, while the other half undergoes charge exchange and rapidly escapes in the form of energetic neutral atoms (e.g. Bagenal and Delamere, 2011). Newly ionized particles are brought to corotation with the magnetic field and form a dense plasma torus around Io's orbit before being transported radially outward in the magnetosphere. Following the tilt of the magnetic dipole of Jupiter, the plasma torus centrifugal equator is tilted relative to the Io orbital plane by $\sim 6.7$ degrees (Gledhill, 1967). As a result, Io is located alternately close to the dense torus center or close to its southern or northern boundaries. Io's orbital period is around $42 \mathrm{~h}$, while plasma torus particles rotate around Jupiter in $\sim 10 \mathrm{~h}$ (Jupiter's rotational period). As a consequence, Io and its conducting ionosphere act as an obstacle to the plasma flow (e.g. Saur et al., 1999). The disturbance and the

\footnotetext{
* Corresponding author. Tel.: +3243669772

E-mail address: b.bonfond@ulg.ac.be (B. Bonfond).
}

related electric currents propagate along magnetic field lines in the form of Alfvén waves (see reviews by Kivelson et al., 2004 and Saur et al., 2004). The combination of the motion of the flux tubes relative to Io and the comparatively low Alfvén speed in the dense plasma torus, causes the paths of these waves, called the Alfvén wings, to be tilted towards the downstream direction in a reference frame fixed with Io. Powerful auroral emissions, called the Io footprints (IFP), are found at the Jovian extremities of these Alfvén wings in each hemisphere (Connerney et al., 1993; Prangé et al., 1996; Clarke et al., 1996).

In each hemisphere, the Io footprint consists of several individual spots followed by a fainter tail extending as far as $100^{\circ}$ of longitude downstream in the corotational direction (Clarke et al., 2002). Recent studies showed that the relative location of these different spots varies with the position of Io in the plasma torus (Gérard et al., 2006; Serio and Clarke, 2008; Bonfond et al., 2008, 2009). Bonfond et al. (2008) distinguished three different spots and associated them with three different mechanisms. The Main Alfvén Wing (MAW) spot is associated with the direct Alfvén wing connecting Io to the Jovian ionosphere. This MAW spot is generally the brightest feature and corresponds to the part of the Alfvern wave's energy that directly escapes the plasma torus. The other 
part undergoes reflections on the latitudinal torus density gradient (Neubauer, 1980; Gurnett and Goertz, 1981). A fraction of these reflected waves can then escape the torus through the opposite boundary, leading to the Reflected Alfvén Wing (RAW) spot. Finally, the Alfvén waves are presumed to accelerate electrons not only directly towards the planet, but also in the opposite direction, in the form of electron beams (Swift, 2007; Jones and Su, 2008; Hess et al., 2010). These beams of electrons cross the plasma torus and eventually precipitate in the atmosphere, generating the Trans-hemispheric Electron Beam (TEB) spot. Wilkinson (1998) offered a similar explanation for the periodicity of the Io-related decametric radio emissions. Additionally, it should be noted that these electron beams have been observed by the Galileo spacecraft as it flew by Io (e.g. Williams and Thorne, 2003) and simulations by Jacobsen et al. (2010) showed that the geometry of the Alfvén wave propagation and the electron beam path are consistent with both the auroral observations at Jupiter and the in-situ observations at Io.

The IFP results from a long chain of processes (see Fig. 1), and various parameters can impact the brightness of the different components of the IFP. This chain of processes along the magnetic field line was first decomposed and modeled by Hess et al. (2010) and is the object of a companion paper (Hess et al., this issue, hereafter Paper II) which addresses the modeling issues related to the observations presented here. It can be summarized as follows. First of all, the power of the electro-magnetic interaction at Io depends on the density of the plasma, which varies as Io moves across the torus. Secondly, the location of Io in the plasma torus also impacts the geometry of the wave propagation and the time required for the waves to cross the plasma torus before they escape towards the poles. This effect could be crucial if a significant damping of these waves occurred within the torus. Another critical point is the reflection ratio by the torus density gradient. Wright (1987) showed that the amount of reflection could be very significant. Hence, Hess et al. (2010) showed that the transmission coefficient could tremendously increase as the scale of the Alfvén waves decreases. Following Chust et al. (2005), they

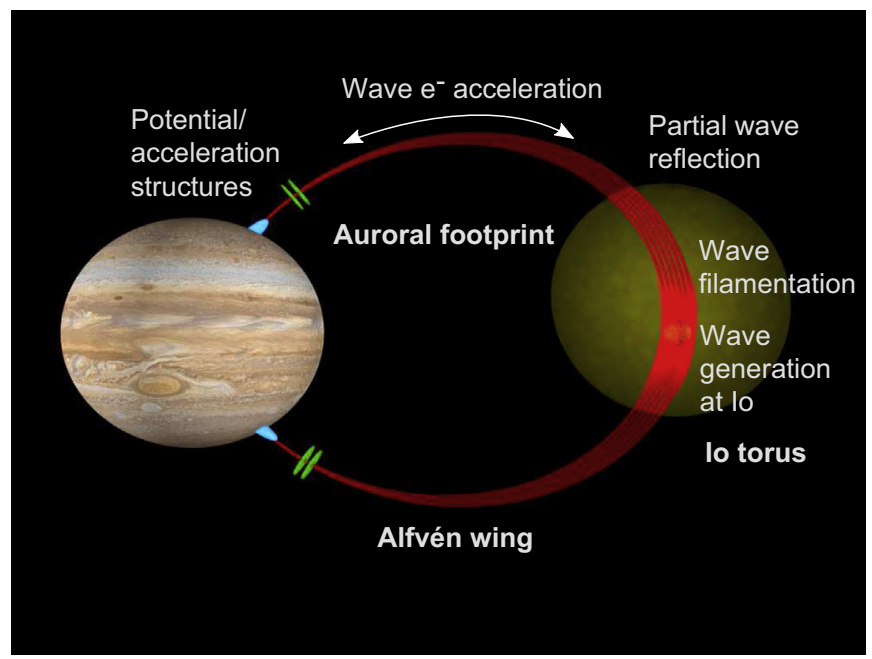

Fig. 1. Scheme of the chain of processes leading to the Io footprint auroral spots. First Alfvén waves are generated close to Io as a result of the interaction between Io and the magnetized plasma of the torus. These waves then propagate towards Jupiter, forming Alfvén wings. They first undergo filamentation, as the large scale waves break into smaller scale structures, before being partially reflected at the torus latitudinal density gradient. Once out of the torus, the Alfvén waves enter the inertial regime and accelerate electrons in both directions along the magnetic field lines. Before they precipitate into the atmosphere and create the auroral footprint the electrons go through additional potential acceleration structures in the Jovian ionosphere. More details about this long chain of processes and how they affect the spots' brightness can be found in Paper II (this issue). argued that only a significant filamentation of the Alfvén waves could explain the observed power of the IFP. The efficiency of the energy transmission between the Alfvén wave and the electron acceleration varies as well, as a function of the magnetic field strength. Finally, the magnetic field strength also controls the size of the loss cone, and thus the amount of electrons that ultimately precipitate into the atmosphere. It is noticeable that all these parameters vary with the System III (S3) longitude of Io, and thus the footprint brightness is expected to be a function of this longitude system. A more detailed study of those processes and of their dependency on Io's S3 longitude can be found in Paper II (this issue).

In the study describing the first detection of the Io footprint, Connerney et al. (1993) suggested that the apparent lack of detection of the infrared (IR) northern IFP in the $90^{\circ}-240^{\circ} \mathrm{S} 3$ longitude range is related to the expected high surface magnetic field strength in this region. Early studies relating the first observations of the UV IFP, observed either with the Faint Object Camera (Prangé et al., 1996, 1998) or the Wide Field and Planetary Camera 2 (WFPC2) (Clarke et al., 1996) on board the Hubble Space Telescope (HST), the datasets were considered too sparse to show any signature of the variations of the footprint brightness as a function of Io's longitude. Subsequent WFPC2 observations demonstrated the existence of a northern footprint in the $90-240^{\circ}$ longitude range (Clarke et al., 1998), contrary to the first results in the IR. The first conclusive relationship between the brightness and Io's longitude was reported by Gérard et al. (2006), based on observations from the Space Telescope Imaging Spectrograph (STIS) on board HST acquired between December 2000 and February 2003. They showed that the maximum IFP brightness increases when Io's centrifugal latitude approaches $0^{\circ}$. The authors attributed the brightening of the IFP as Io settles into the dense torus center to the expected enhancement of the Io-magnetosphere interaction. Serio and Clarke (2008) studied the evolution of the footprint UV brightness on STIS images acquired from August 1999 to January 2001. They concluded, like Gérard et al. (2006), that the energy radiated away from the local interaction at Io, which is controlled by the plasma torus density at the satellite, is the main driver for the mean IFP brightness. Wannawichian et al. (2010) performed a similar study, but included a much larger dataset acquired with the Advanced Camera for Surveys (ACS) onboard HST. They also observed two brightness peaks, at $110^{\circ}$ Io SIII longitude and $290^{\circ}$ Io SIII longitude, i.e. when Io is close to the torus center, and reached the same conclusion. The previously reported IFP brightness and emitted powers are summarized in Table 1 . The variations of the energy fluxes generated at Io as a function of the Io S3 longitude have been recently studied by Saur et al. (2013) and Wannawichian et al. (in press). However, the local variations may not be sufficient

Table 1

List of the published emitted and electron precipitated power for the FUV Io footprint. For Gérard et al. (2006), the printed value is $0.4-8 \mathrm{GW}$ but, after verification, it appears that this 10 times smaller value came from a typo in a conversion coefficient. We thus consider here the corrected value.

\begin{tabular}{llll}
\hline & $\begin{array}{l}\text { Emitted power } \\
(\mathrm{W})\end{array}$ & $\begin{array}{l}\text { Input power } \\
(\mathrm{W})\end{array}$ & $\begin{array}{l}\text { Brightness } \\
(\mathrm{kR})\end{array}$ \\
\hline $\begin{array}{lll}\text { Prangé et al. (1996) } \\
\text { Clarke et al. (1996) }\end{array}$ & $5 \times 10^{10}$ & $2-3 \times 10^{11}$ & 700 \\
$\begin{array}{l}\text { Prangé et al. (1998) } \\
\text { Clarke et al. (1998) }\end{array}$ & $10^{11}$ & $60-120$ \\
$\begin{array}{l}\text { Gérard et al. (2006) } \\
\text { Serio and Clarke (2008) }\end{array}$ & $0.8-5 \times 10^{11}$ & $35-250$ \\
Wannawichian et al. & & $0.4-8 \times 10^{10}$ & $25-220$ \\
(2010) & & $40-480$ \\
This study & & & $5-390$ \\
\hline
\end{tabular}

${ }^{1}$ These numbers are for the MAW spot only. 
to explain the detailed fluctuations of the spots' brightness, and effects of the far field likely play an important role as well, as we will further show below and in Paper II.

The relative motion of the various spots strongly suggests that they arise from different mechanisms, and that the parameters controlling their brightness could be different. Bonfond (2010) showed that the mean electron energy for the MAW and the TEB spots is different since the altitude of the TEB spot is $200 \mathrm{~km}$ lower than the altitude of the MAW spot. Therefore, here we consider the different components of the UV Io footprint individually when measuring their brightness. Finally, Bonfond et al. (2007) found that, on top of the System III related brightness variations, the MAW and the TEB spot brightness fluctuates by $~ 30 \%$ (and up to $50 \%$ ) on timescales of minutes. We include in the present study STIS observations from 2009 confirming this finding. Moreover, current high quality datasets encompass more than 10 years of observations, making long term comparisons possible. We will thus discuss brightness variations and their possible causes on all these different timescales.

\section{Data processing}

\subsection{Dataset, limb fitting and background removal}

The present study makes use of the entire dataset of Far-UV $(\sim 115-170 \mathrm{~nm})$ high resolution and high sensitivity images acquired with HST/STIS and ACS instruments from 1997 to 2009. The Far-UV channel of both instruments uses the same type of Multi Anode Micro-Channel Array (MAMA) detector and is called FUV-MAMA on STIS and Solar Blind Channel (SBC) on ACS. STIS/ FUV-MAMA has a slightly better spatial sampling than ACS/SBC $(0.025 \times 0.025$ arcsec per pixel compared to $0.030 \times 0.034$ arcsec per pixel). At the same time, ACS/SBC is three to four times more sensitive than STIS/FUV-MAMA, depending on the wavelength, but it is affected by a stronger "red leak". Indeed, the longer wavelength emissions, such as the reflected solar light, are much less effectively cut off, which results in a brighter planetary background on the ACS images.

The Jovian auroral emissions in the UV domain result from the impact of electrons on $\mathrm{H}_{2}$ molecules and $\mathrm{H}$ atoms. HST Images of the Jovian aurora have been acquired with two different filters for each instrument, i.e. the CLEAR (25MAMA) and the Strontium Fluoride (F25SrF2) filters for STIS and the F115LP and the F125LP filters for ACS. The CLEAR and the F115LP filters include the $L y-\alpha$ atomic $H$ line while the Strontium Fluoride and the F125LP transmissions sharply drop just before this line, thus avoiding contamination from geocoronal emissions. In order to compare brightness on images acquired with these different filters, we used the conversion coefficients from Gustin et al. (2012) and we assumed a typical IFP color ratio of 1.8 (Gérard et al., 2002). The values provided in the present study are either the brightness or the emitted power by $\mathrm{H}_{2}$ molecules in the $70-180 \mathrm{~nm}$ wavelength range. It should be noted that previously published values only accounted for the Far-UV part of the $\mathrm{H}_{2}$ spectrum, which represents approximately half of the total emissions. This partly explains why the values reported in the present paper are larger than some published previously.

The STIS/FUV-MAMA has two acquisition modes and both of them have been used in this study. The first one is the ACCUM mode, for which the photon detection events are accumulated in each pixel during a given exposure time in order to form an image. The second one is the TIMETAG mode, which provides events lists containing the location and arrival time of each detected photon. The events lists can be subsequently used to create image sequences at very high time resolution, providing that the signal to noise ratio is sufficient. Only the ACCUM mode is available on the other instrument considered in the present study, i.e. the ACS/SBC.

As detailed below, the methods we developed require one to precisely locate the different IFP spots on the planet, which demands knowledge of the location of the planetary center on the images. Unfortunately, the pointing accuracy of the guide star acquisition mode used for these observations is not sufficient for that purpose. As a consequence, Jupiter's center position is determined using the limb fitting method described by Bonfond (2009).

Even if the polar regions appear darker than the rest of the planetary disk, this background emission still has to be removed before the actual brightness of the aurora can be measured. We first removed an empirically built planetary disk (Bonfond et al. 2011). For more accuracy, we then subtracted from the resulting image the mean brightness measured in two 20-pixel wide squares located $5^{\circ}$ upstream and $5^{\circ}$ equatorward of the MAW spot, respectively.

\subsection{Estimates of the auroral brightness}

Most studies discussing the intensity of the auroral features specify brightnesses in units of Rayleighs or kilo-Rayleighs. However, the Rayleigh $(R)$ is a very peculiar unit that should be handled with care. This radiance unit measures the absolute angular surface brightness of spatially extended light emitting sources (Baker, 1974). $1 \mathrm{kR}$ represents $10^{9}$ photons $\mathrm{cm}^{-2} \mathrm{~s}^{-1}$ emitted within $4 \pi$ steradians. This unit was initially used for airglow emissions on Earth but it is also used for auroral emissions, since brightness measurements account for the light emissions integrated all along the vertical air column. The brightness expressed in $\mathrm{kR}$ can then be related to the precipitated energy flux through the use of appropriate energy degradation models (Gérard and Singh, 1982). However, such a conversion requires knowledge of the vertically integrated brightness. This quantity is not trivial to derive from HST images, since the aurora is always observed sideways rather than directly from nadir or from zenith. On HST images of giant planet aurorae, emissions appearing close to the limb usually look brighter than those found closer to the planetary center. This limb brightening is caused by the geometrical accumulation of optically thin emissions along the line of sight. This effect can increase the observed brightness by one order of magnitude (Grodent et al., 1997). As a result, previous works made use of different strategies to circumvent this issue. In order to compare images with different observation geometries, Clarke et al. (1998) applied the plane parallel correction to convert the apparent brightness to the brightness that would be expected if the IFP was located on the central meridian at $67^{\circ}$ latitude. The plane parallel correction assumes that the atmosphere can be modeled as a flat emitting layer. If $\alpha$ is the zenith angle, i.e. the angle between the observer and the normal to the plane, than the correction coefficient to convert the observed brightness into the brightness that an observer looking at the nadir would see is simply $\sec (\alpha)$. Gérard et al. (2006) used the same assumption to compensate for the limb brightening, but provided a corrected value for an observer looking at nadir. Alternatively, the correction factor provided by Serio and Clarke (2008) assumed the emission region to be an extended emitting shell instead of a flat layer. The optical depth correction factors corresponding to these different assumptions are shown in Fig. 2. In this figure, the vertical dash-dotted line represents the minimum distance to the limb below which Serio and Clarke (2008) consider their correction unreliable. We note that above this value, differences with the plane parallel correction are barely noticeable, so that the methods used in these different studies are equivalent in practice. The corrected brightness in Serio 

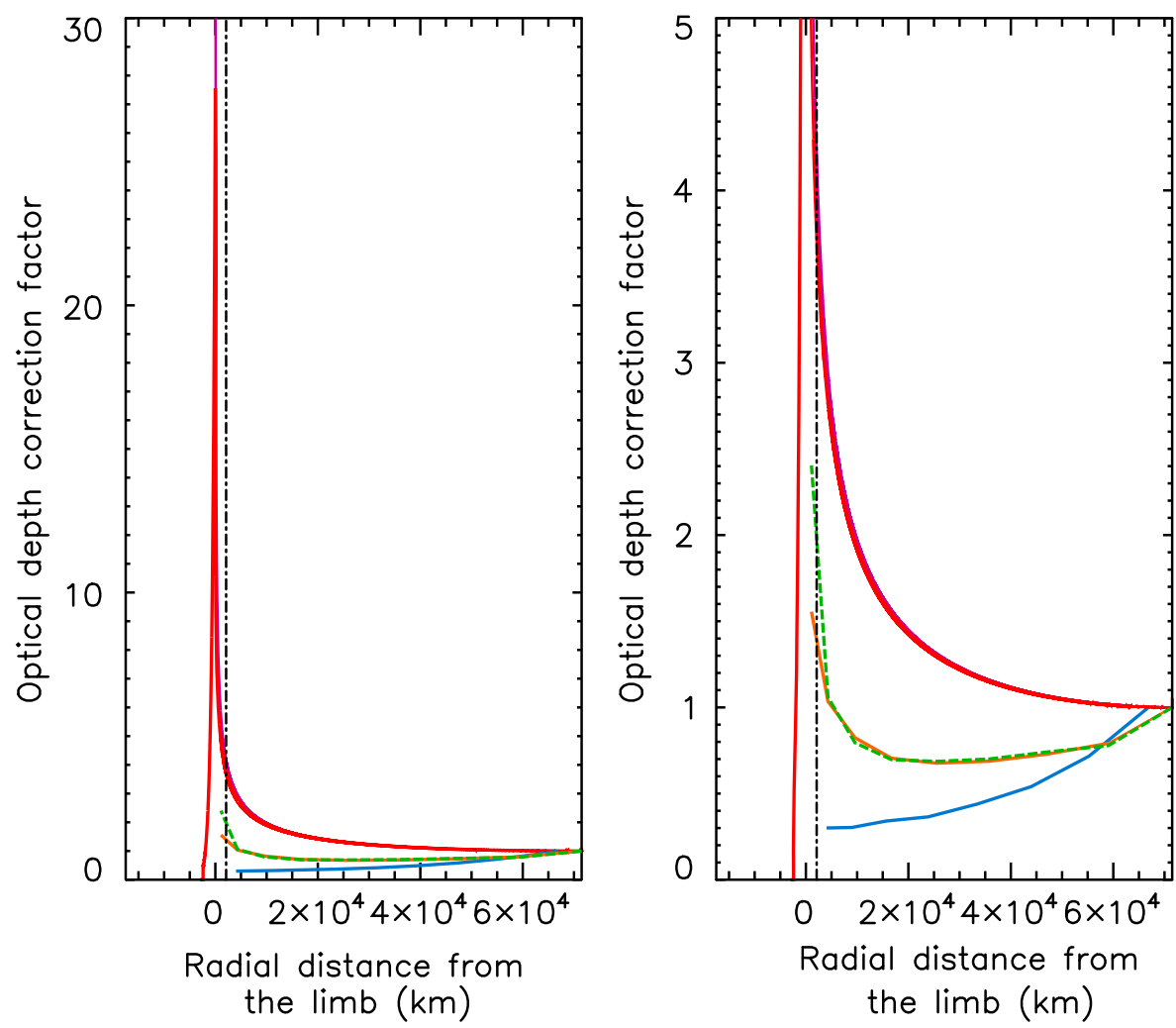

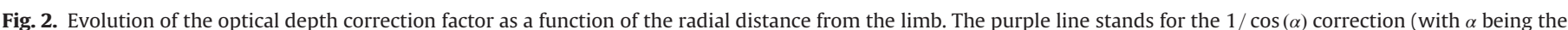

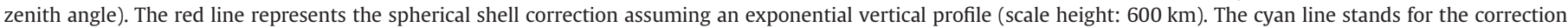

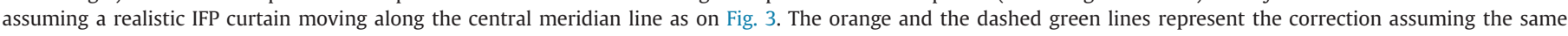

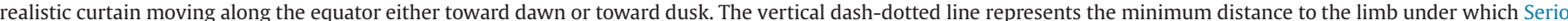

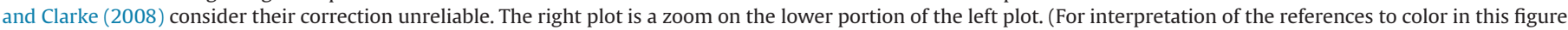
caption, the reader is referred to the web version of this article.)

and Clarke (2008) assumes a footprint located on the central meridian at $67^{\circ}$ latitude similarly to Clarke et al. (1998). Finally, Wannawichian et al. (2010) presented both uncorrected and corrected brightness averaged over a $0.25 \times 0.25 \operatorname{arcsec}^{2}$ square, using the same correction method as Serio and Clarke (2008).

All correction methods mentioned above implicitly assume that the horizontal extent of the IFP is large. However, this assumption is not verified since images of the IFP, when observed from the side, show that the IFP is a thin curtain no broader than $200 \mathrm{~km}$ (Bonfond, 2010). The evolution of the apparent brightness for different positions of the Io footprint on the planet is thus much more complex, as illustrated by Fig. 3. This figure shows simulated images of hypothetical footprints consisting of a $900 \times 200 \mathrm{~km}$ wide single spot followed by an extended tail. The brightness of this tail falls off exponentially with an e-folding distance of $20,000 \mathrm{~km}$. The spatial extent and the total power is exactly the same for each footprint. In each case, the spot and the tail are extending along a parallel of latitude. This geometry was simulated for 17 equally spaced $\left(5^{\circ}\right)$ parallels from $0^{\circ}$ to $85^{\circ}$. We note that the maximum brightness of the spot decreases as it reaches higher latitudes and thus approaches the limb, contrary to the behavior expected from limb brightening of an extended emitting area. Nevertheless, tail emissions show that some limb brightening actually takes place and increases the apparent brightness as the light accumulates along the line of sight.

In conclusion, no simple correction can be applied to infer the vertical brightness from the apparent one. All the complexity of the observing geometry has to be accounted for to retrieve information on the precipitated energy fluxes. As a result, we propose a new method to estimate the vertical brightness of the IFP spots and tail. It consists of comparing the emissions observed with the HST STIS and ACS instruments with realistic simulations of the emission regions. This method requires the IFP auroral curtain to be as perpendicular to the line of sight as possible and to be away from other auroral emissions. These constraints do not pose any particular issue in the southern hemisphere, as long as the MAW spot remains $\leq 30^{\circ}$ away from the central meridian line. However, the larger offset of the northern oval and the non-circular shape of the Io footpath prevents our method from disentangling the different spots outside the $100-300^{\circ}$ Io SIII longitude range.

\subsection{Determination of the PSFs}

Before being compared with actual observations, the simulated images have to be convolved with the appropriate instrumental Point Spread Function (PSF). In this section, we describe how these PSFs have been determined. Since the shape of a point source varies with the instrument and the filter, we need 4 different PSFs. Moreover, the PSF is usually dependent on the position of the object on the detector. This is particularly critical for the ACS (Pavlovsky et al., 2006). For this instrument, most images of any given hemisphere have been acquired with the same orientation. The Io footprint is thus usually located in the same area on the images, depending on the hemisphere. Two PSFs per filter, one per hemisphere, are thus necessary. However for STIS, only one PSF per filter was determined, since geometric distortions are less critical than for ACS.

The PSFs necessary for this work were determined using an algorithm based on the non-violation of the sampling theorem (Shannon, 1949). It allows one to fit a PSF to several point sources present in the same frame. It starts from an approximate PSF and adds a numerical background to it, so that the original image is 


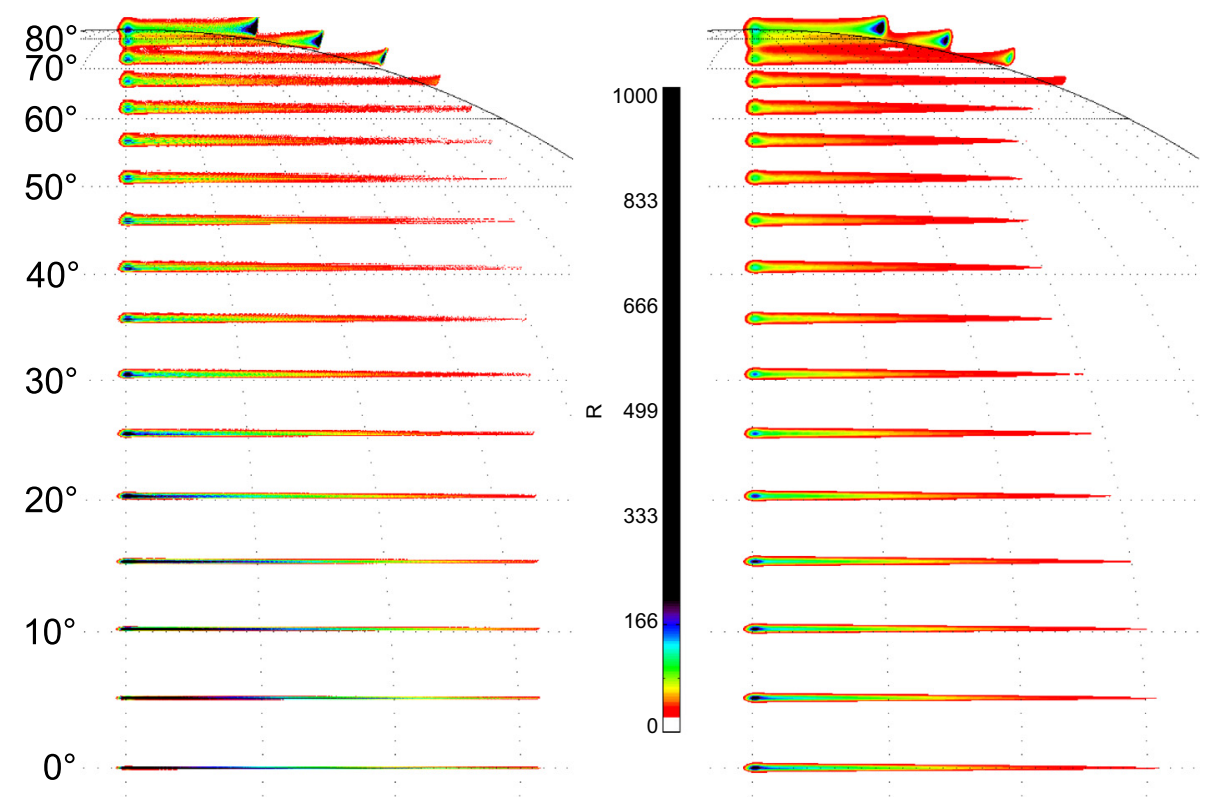

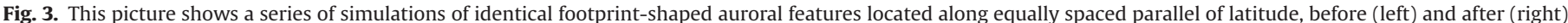

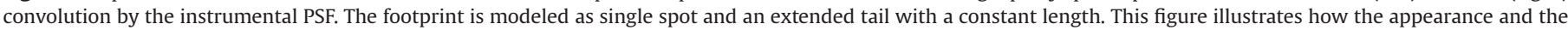

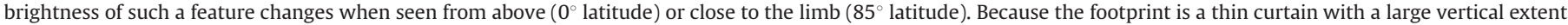

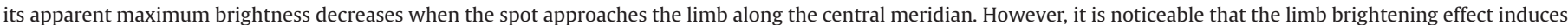
an apparent brightness enhancement in the tail.

well reproduced in terms of $\chi^{2}$. The intensities and positions of the point sources are adjusted through the process. For the interested readers, the algorithm is explained in detail in Magain et al. (2007).

To actually determine the PSFs, we retrieved HST archival data of the globular cluster NGC 6681, through the appropriate filters. NGC 6681 provides a well-distributed field permitting a satisfying determination of the PSF. For each IFP mean position on the detector, each filter and each instrument, four stars were selected in the zone of interest. For each case, a first guess of the PSF was computed using the Tiny Tim software (Krist and Hook, 2004). This initial PSF was then fitted to the four selected stars with the algorithm described here above. While the Full Width at Half Maximum (FWHM) of the Tiny-Tim PSF lies around 1-2 pixels, the FWHM of the final PSF lies between 2 and 3 pixels.

All simulated footprints in Fig. 3 are characterized by the same total emitted power, whatever the latitude. The left column corresponds to un-convolved images, while the right images have been convolved with the STIS CLEAR PSF. If one selects a reasonable area (e.g. a 10-pixel wide square) on the images to isolate the spot at the footprint head, the number of counts in this area would be much lower in the convolved image than in the un-convolved one. The present study realistically accounts for this effect, which is another reason why our estimates of the emitted power are higher than those derived in some previous studies.

\section{Emission model}

The purpose of our emission model is to reproduce as accurately as possible the shape and brightness of the different components of the Io footprint in order to compare model images to the actual images after planetary disk subtraction. For each HST image, four model images are computed, one for each of the three spots and one for the tail. Each sub-image is built in such a way that the maximum vertical brightness is $1 \mathrm{kR}$. The final simulated image is composed of a linear combination of these sub-images. We consequently compute the set of coefficients which provides the linear combination of the sub-images best matching the observed image, in the sense of the least squares. This set of multiplicative coefficients represent the maximum vertical brightness of the different spots and the tail.

All four sub-structures have been observed to be aligned along reference footpaths (one per hemisphere) fixed in System III (Bonfond et al., 2009). The relative position of the simulated spots is allowed to vary as a function of Io's S3 longitude, according to the inter-spot distances reported by the same authors. For spot $s$, the vertically integrated brightness is assumed to vary along and across the footpath following a 2D Gaussian law:

$I_{S}(x, y)=e^{\left(-\frac{\left(x-l o n_{s}\right)^{2}}{2 \sigma_{\sigma_{s} n_{s}}^{2}}-\frac{(y)^{2}}{2 \sigma_{\text {lats }}^{2}}\right)}$

where $x$ is the distance along the footpath, $y$ is the distance perpendicular to the footpath, $\operatorname{lon}_{s}$ is the position of the spot's brightness maximum along the footpath, $\sigma_{l o n_{\mathrm{s}}}$ characterizes the spot width along the footpath and $\sigma_{\text {lat }_{s}}$ characterizes the spot width perpendicular to the footpath.

As far as the tail is concerned, the brightness profile perpendicular to the footpath is described by a Gaussian function as well, but the evolution of the brightness along the footpath is described by an empirical function which starts as a half Gaussian law and then evolves into a decreasing exponential law. This formulation makes it possible to easily adjust all the relevant parameters, such as the position of the maximum and the e-folding distance:

$I_{\text {trail }}(i, j)=a_{t}\left(i-d_{\text {lon }}\right)^{n_{t}} e^{\left(-b_{t} *\left(i-d_{l o n}\right)-\frac{(j)^{2}}{2 \sigma_{\text {lat }}^{2}}\right)}$

with

$a_{t}=\left(\frac{1}{\operatorname{lon}_{t}}\right)^{n_{t}} e^{n_{t}}$

$b_{t}=\frac{n_{t}}{\operatorname{lon}_{t}}$ 
$n_{t}=\frac{e^{1}}{\left(\frac{H}{\operatorname{lon}_{t}}\right)-\ln \left(\frac{H}{\operatorname{lon}_{t}}\right)-1}$

and where $H$ is the e-folding distance, $\operatorname{lon}_{t}$ is the position of the tail maximum along the footpath which is set to $\operatorname{lon}_{\text {MAW }}+\sigma_{\operatorname{lon}_{\text {MAW }}}$ and $d_{l o n}$ is the tail starting position along the footpath, which is set to $\operatorname{lon}_{\text {MAW }}-\sigma_{\operatorname{lon}_{\text {MAW }}}$.

The brightness in the above equations corresponds to the vertically integrated brightness, which is the relevant parameter to derive the incoming particle flux. However, to compute the model images corresponding to an oblique observation, the vertical emission rate profile has to be specified and is described with a Chapman profile of the form:

$f(x)=C \exp \left(1-\frac{Z-Z_{0}}{H}-\exp \left(-\frac{Z-Z_{0}}{H}\right)\right)$

where $C$ is a constant, $Z$ is the altitude, $Z_{0}$ is the altitude of the peak and $H$ is the scale height in $\mathrm{km}$. The parallel and perpendicular sizes of the different sub-structures as well as their peak altitude and their vertical scale height are gathered in Table 2 .

At this stage of the modeling process, we have determined the longitude, the latitude, the altitude and emission rates of the emission points corresponding to the different spots and the tail. The spatial coordinates of the emission regions are converted into $x-y-z$ coordinates related to the HST image, $x$ and $y$ corresponding to the image abscissae and ordinates and $z$ corresponding to the depth. For each $x-y$ couple, the emissions are integrated along the $z$ direction to produce the pixel brightness. In order to limit the spatial sampling error, the side of the voxels (i.e. the volume elements in the simulation) is half the size of the pixel side. The final images are thus twice as large as the observations and are subsequently re-binned to the original pixel size. One model image is produced for each sub-structure, and this image is then convolved with the PSF corresponding to the relevant instrument, filter and footprint location on the detector.

Because of the finite accuracy of the reference footpath, the fine tuning of the simulated images' position is computed by adjusting the barycentre of the MAW spot (using the brightness as a weighting factor) to the one of the original image after background removal. The final step is a least squares fit of the relative brightnesses of the different IFP features to the observed image. This over-determined problem was solved with the singular values decomposition (SVD) method. The singular values are systematically significantly above 0 , but they usually decrease as the MAW and the TEB begin to overlap. In a few cases, as the RAW or the TEB spots brightness get very weak, the SVD method provides negative values for the brightness of these spots; a solution that is not physically acceptable. In these cases, an iterative LevenbergMarquardt least square fit method, imposing the non-negativity of the solution, is used instead. In any case, the fitting coefficients provide the maximum vertical brightness for each sub-structure in $\mathrm{kR}$.

\section{Table 2}

Size parameters for the simulated spots and tail. The relationship between the $\sigma$ parameter of a Gaussian and its full width at half maximum (FWHM) is: $F W H M=2 \sqrt{2 \ln 2} \sigma$.

\begin{tabular}{lllll}
\hline & $\begin{array}{l}F W H M_{\text {lon }} \text { or } \\
\text { e-folding distance }\end{array}$ & FWHW & $\begin{array}{l}\text { Vertical } \\
\text { scale height }\end{array}$ & $\begin{array}{l}\text { Peak } \\
\text { altitude }\end{array}$ \\
\hline MAW spot & $900 \mathrm{~km}$ & $200 \mathrm{~km}$ & $400 \mathrm{~km}$ & $900 \mathrm{~km}$ \\
TEB spot & $900 \mathrm{~km}$ & $200 \mathrm{~km}$ & $200 \mathrm{~km}$ & $700 \mathrm{~km}$ \\
RAW spot & $900 \mathrm{~km}$ & $200 \mathrm{~km}$ & $400 \mathrm{~km}$ & $900 \mathrm{~km}$ \\
Tail & $20,000 \mathrm{~km}$ & $200 \mathrm{~km}$ & $400 \mathrm{~km}$ & $900 \mathrm{~km}$ \\
\hline
\end{tabular}

The description of the initial part of the tail is a major limitation of our method. As far as the beginning of the tail is concerned, we chose a reasonable empirical formulation of the brightness along the footpath because current models of the electro-magnetic interaction focus either on the initial phase of the interaction, leading to the spots, or on the tail when it is well established, but not specifically on the transition phase (Phase 2 in the Delamere et al., 2003 formulation).

In the present study, the error bars on the modeled emitted power account for the sum of two uncertainty sources. The first one is the counting uncertainty in the photon detection events. Considering the count rate as a Poissonian process, this uncertainty is computed as the standard deviation of the count rate over the exposure time before the background removal. It accounts for approximately $40 \%$ of the uncertainty estimate. The second and largest source of uncertainty is caused by the imperfect shape and location of the spots in the simulation. It is computed as the square root of the quadratic sum of the count rate difference between the HST image and the simulated image in each spot area.

Here above, we described our measurement method based on 3D simulations of the different features of the IFP. The next section is dedicated to the observational results, focussing first on the variations of the brightness with the S3 longitude of Io. In particular, we compare the different spots and the different hemispheres. We then discuss brightness variation on timescales of minutes and on timescales of years.

\section{Results and discussion}

\subsection{The various components of the IFP}

One of the main advantages of the brightness units over the power units is the direct connection between the vertical brightness and the precipitating energy fluxes. However, in the case of HST observations of the Jovian polar regions, the angle between the local zenith and the line of sight is far from $0^{\circ}$. If the emission region is an extended area, the observed brightness can be easily converted into vertical brightness by means of the plane parallel or the spherical shell approximations. Unfortunately, measurements of the IFP size and shape demonstrate that the Io footprint forms a thin curtain made of different spots and a tail, and that it cannot be considered as an extended area (Bonfond, 2010). Fig. 2 shows that values computed with any correction method based on the extended area assumption are much smaller than the observed brightness, while a nadir-looking observer would actually measure larger values than HST. Fig. 4 shows the maximum brightness of the MAW spot (i.e. the brightness of the brightest pixel) as a function of the S3 longitude of Io without any correction. The global trend seems to follow the previous conclusions from Gérard et al. (2006), Serio and Clarke (2008) and Wannawichian et al. (2010). The maximum MAW spot brightness is observed when Io lies around $110^{\circ} \mathrm{S} 3$ longitude, and a possible second peak appears around $290^{\circ}$ for the northern IFP, which corresponds to the region where Io is near the torus center. The main difference lies in the brightness values, which now account for the whole Extreme-UV $(80-120 \mathrm{~nm})$ and Far-UV $(120-180 \mathrm{~nm}) \mathrm{H}_{2}$ spectrum and are not reduced by any limb brightening correction.

Our new measurement method addresses a different point of view and tries to answer the following question: what is the maximum vertical brightness of the different spots and the tail best matching the observations? The results for the MAW spot are shown in Fig. 5.

First, we note that the plotted values are larger than earlier estimates (see Table 1). The first reason for this difference stems from the fact that the maximum brightness as observed from 
above is higher than seen from the side, since the vertical scale height is assumed to be $\sim 400 \mathrm{~km}$ while the width is only $\sim 200 \mathrm{~km}$. The second reason is related to the point spread function (PSF). Bonfond (2010) noted that the PSF does not significantly affect the position and the size of the different features of the IFP because its FWHM is $\sim 2-3$ pixels. However, as far as the maximum brightness is concerned, the impact of the PSF cannot be neglected because a significant part of the brightness spreads over the PSF wings.

An incoming electron energy flux of $1 \mathrm{~mW} / \mathrm{m}^{2}$ results in a vertical brightness of $\sim 10 \mathrm{kR}$ of $\mathrm{H}_{2}$ UV emissions over a wide range of energy of the primary electrons (Gustin et al., 2012). The mean photon energy in the $H_{2}$ UV spectrum is $\sim 1.8 \times 10^{-18} \mathrm{~J}(11.2 \mathrm{eV})$. Consequently, $10 \mathrm{kR}$ correspond to an emitted flux of $0.18 \mathrm{~mW} / \mathrm{m}^{2}$ and the ratio between the precipitated and the emitted power lies around $20 \%$. We find that the incoming flux for the MAW spot ranges between 250 and $2000 \mathrm{~mW} / \mathrm{m}^{2}$. As noted by Prangé et al.

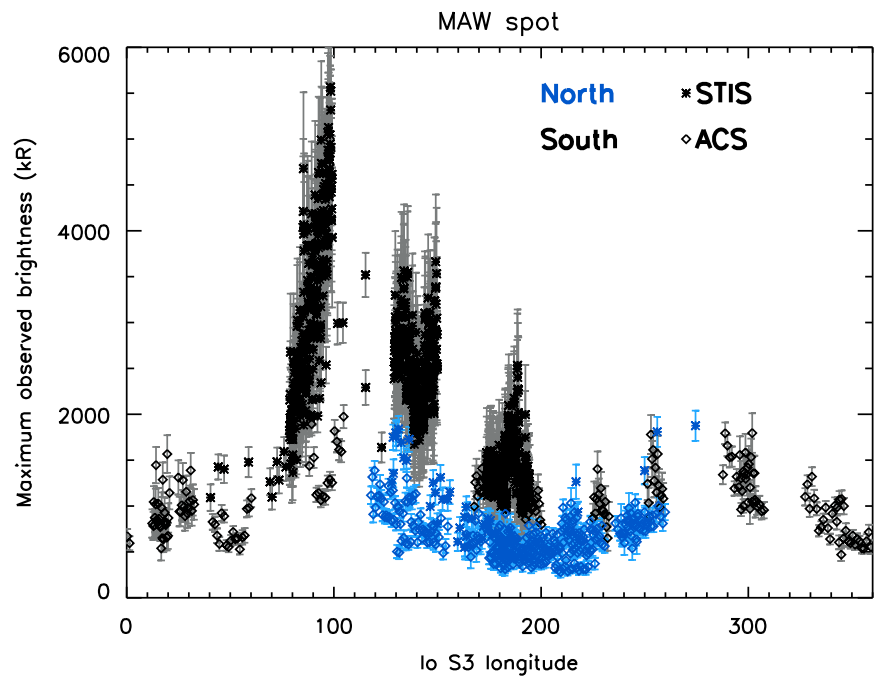

Fig. 4. Evolution of the maximum observed brightness of the MAW spot as a function of the Io longitude. Black symbols are related to the southern hemisphere while blue symbols are related to the northern hemisphere. The star symbols represent STIS observations while the diamond symbols represent ACS observations. The error bars account for the photon counting uncertainty on the pixel of interest. No limb brightening correction was applied to the plotted values.

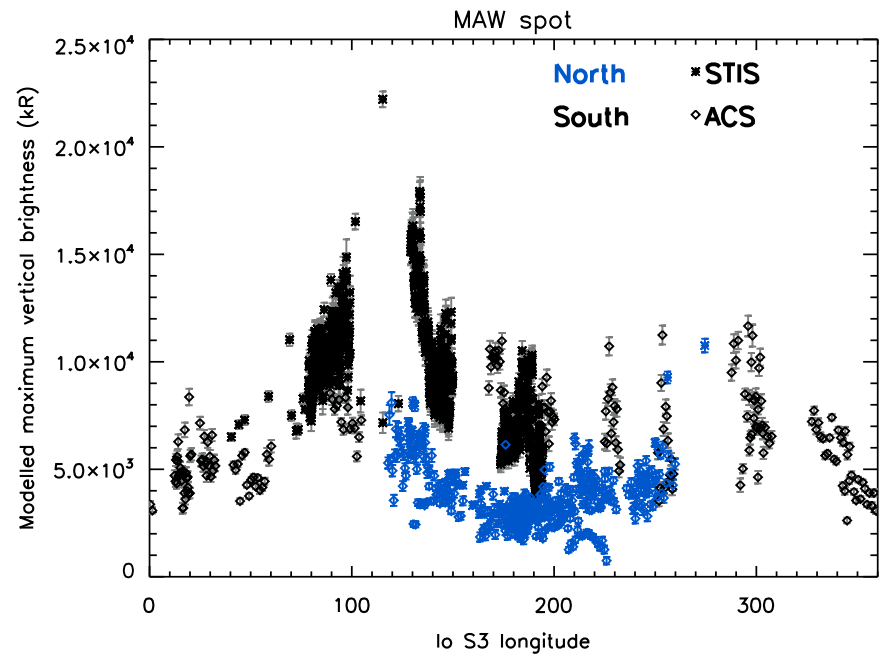

Fig. 5. Evolution of the modeled maximum vertical brightness as a function of the Io longitude. Black symbols are related to the southern hemisphere while blue symbols are related to the northern hemisphere. The star symbols represent STIS observations while the diamond symbols represent ACS observations. (For interpretation of the references to color in this figure caption, the reader is referred to the web version of this article.)
(1996), the presence of such high values raises the issue of the atmospheric response to such a sudden (the footprint moves relative to the atmosphere) and localized energy supply.

Figs. 6 and 7 show the emitted power for the MAW, TEB and RAW spots. The brightness of the different spots broadly follows a similar scheme, with a minimum when Io is close to the northern torus boundary. In the southern hemisphere, the brightness of all the spots peaks around $110^{\circ}$, i.e. when Io reaches the torus center. However, around $290^{\circ}$, Io is also close to the torus center, but the brightness barely increases. This is an important observation since it shows that the Io centrifugal latitude, i.e. its position in the torus, is not the only parameter controlling the power of the spots. Moreover, the power of the different spots seems highly correlated, but the relative brightness of the spots evolves significantly. A likely interpretation for this behavior is that the root cause for these different spots is the same, although the detailed mechanism

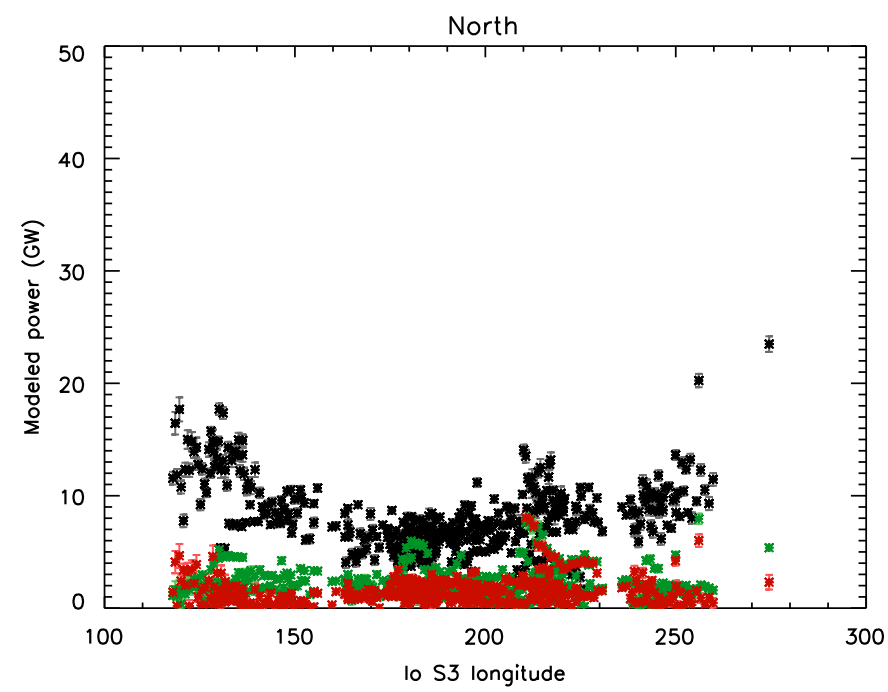

Fig. 6. Evolution of the emitted power of the different IFP spots in the northern hemisphere as a function of the $\mathrm{S} 3$ longitude of Io. The MAW spot emitted power is shown in black, the TEB spot is shown in red and the RAW spot is shown in green (For interpretation of the references to color in this figure caption, the reader is referred to the web version of this article.)

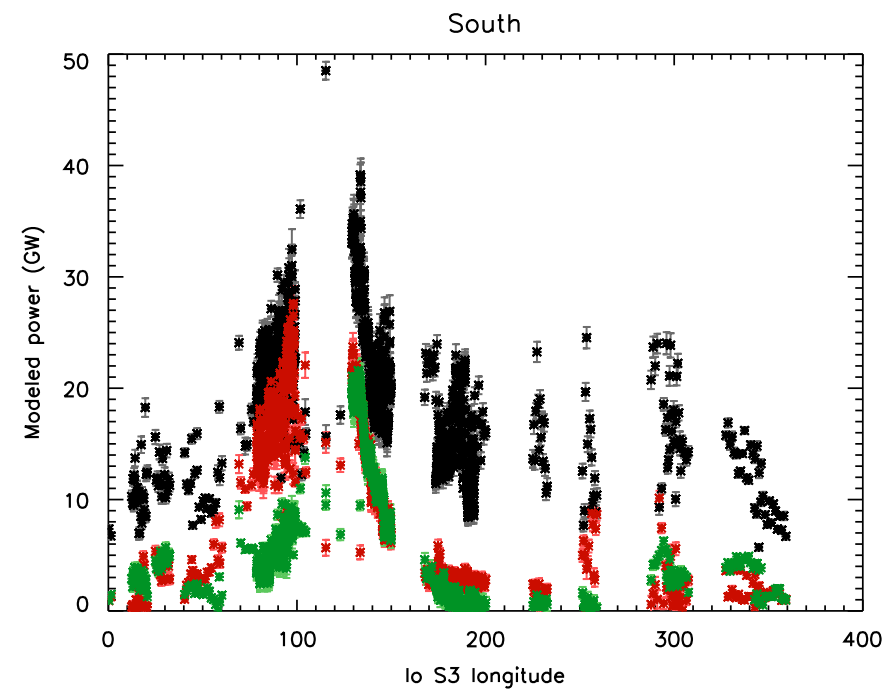

Fig. 7. Evolution of the emitted power of the different IFP spots in the southern hemisphere as a function of the S3 longitude of Io. The MAW spot emitted power is shown in black, the TEB spot is shown in red and the RAW spot is shown in green. (For interpretation of the references to color in this figure caption, the reader is referred to the web version of this article.) 
ultimately leading to each of these spots is different. A more detailed discussion of theoretical implications of these observations can be found in Paper II.

Additionally, we notice that the power emitted by the tail within its first $21,000 \mathrm{~km}$ (the e-folding distance) is twice to $\sim 2.5$ higher than the sum of the three spots.

In conclusion, we find much higher ( $>100$ times) precipitated energy fluxes than previous studies as we account more realistically for the geometry of the Io footprint. Moreover, the ratio of the different spots' brightnesses is not constant with the longitude, even if their brightnesses are highly correlated. Finally, the southern MAW spot brightness is not identical when Io is at $110^{\circ}$ and at $290^{\circ}$, while Io is close to the torus equator in both cases. In the next section, we further investigate the variations of the brightness with the longitude by comparing the emissions from the two hemispheres.

\subsection{North-south comparison}

Figs. 4 and 5 show significant differences between the northern and the southern MAW spots' brightness in the Io S3 longitude range between $150^{\circ}$ and $200^{\circ}$. In this sector, Io is progressively moving from the torus center to the northern torus boundary. Fig. 8 shows the emitted power measured during three particular HST orbits during which both hemispheres have been observed. For the first orbit, the telescope was first pointing to the south pole during the first half of the orbit and then slewed to the north pole. The two other orbits began with North IFP observations and then the field of view shifted towards the South. These cases are very interesting because no long term variation of the torus density can be invoked to explain the observed differences between hemispheres. Moreover, the different spots are well separated, so that their mixing cannot explain the spots' brightness variations in this sector.

The implication of these results are discussed in detail in Paper II, however, here is a summary of the arguments. If the intensity of the electro-magnetic interaction at Io was the only parameter controlling the spots' brightness, then the spots in both hemispheres would have the same brightness. A scenario in which the Alfvén waves are damped as they cross the plasma torus could not explain this difference, since the northern MAW spot should then be brighter than the southern one. Since Io has positive centrifugal latitudes in this sector, the southward Alfvén waves travel longer in the torus than the northward ones. Consequently, the southern MAW spot should be weaker than the northern one, contrary to observations.

A possible reason for a brighter MAW spot in the south is the more efficient transmission of the Alfvén waves through the southern torus boundary than through the northern one, as Io is northward of the centrifugal equator (see Paper II in the present issue for details). However, such an explanation would lead to a weaker TEB spot in the southern hemisphere than in the north, also contrary to observations.

Finally, the brighter MAW spot (and TEB spot, to a lesser extent) in the south could be related to the weaker surface magnetic field, and thus the more open loss cone, in this hemisphere for this longitude range. This mechanism could qualitatively explain our results, however simulations predict that the loss cone effect could not cause such dramatic brightness variations from one hemisphere to another (see Paper II for details).

As a conclusion, observations show that the brightness of the spots is not controlled by the location of Io in the torus only and magnetic field asymmetries certainly play a role, but the precise mechanism remains to be fully uncovered. The timescales of the variations discussed here are of a few hours and are clearly related

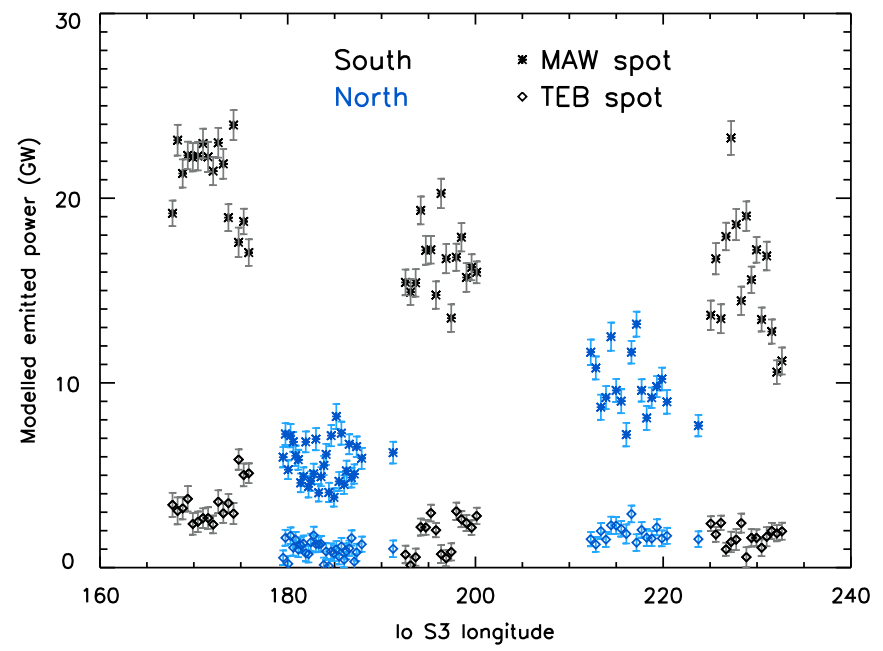

Fig. 8. Evolution of the MAW spot (star symbols) and the TEB spot (diamond symbols) emitted power during three orbits for which hemisphere transition was performed. The first orbit started in the southern hemisphere and ended in the southern hemisphere. On the other hand, the two other orbits began in the north and then transitioned to the south. In the $180-200^{\circ}$ sector, the larger measurement density is due to the superposition of points from the first two orbits. The black symbols represent observations in the southern hemisphere and the blue symbols represent observations in the northern hemisphere. The spot emissions are weaker in the North than in the South, which could be attributed to the difference of surface magnetic field strength. (For interpretation of the references to color in this figure caption, the reader is referred to the web version of this article.)

to the S3 longitude of Io. In the next sections, we focus on shorter (minutes) and longer (years) timescales.

\subsection{Short timescale variations}

Based on a dataset of all TIMETAG STIS images acquired between 1997 and 2003, Bonfond et al. (2007) found that the brightness of the MAW spot systematically showed variations on the order of $30 \%$ with a typical growth time of one minute. However, these sequences were only 2-5 min long, which prevented them from being used to study the possible quasi-periodicity of these variations. In four cases, the southern TEB spot was bright enough to perform a similar analysis. In three cases out of these four, the variations of the two spots were correlated. The limited length of the observation sequences and the limited number of cases for simultaneous variations called for further investigations. Therefore, a HST campaign consisting three observation orbits was carried out in late Summer 2009 in order to study the short timescale variations of the southern Io spots on longer STIS timetag sequences.

Fig. 9 shows the short-timescale power variations for the MAW and the TEB spots. As for the previous observations, strong fluctuations are systematically observed, with a typical time interval of 2-4 min between brightness maxima. The fast fluctuations of the two spots are usually not correlated except in the $78^{\circ}-94^{\circ}$ sector. In this sector, Io comes from the southern torus boundary and approaches the torus center. The MAW and the TEB spots are well separated and the TEB spot begins to get nearly as bright as the MAW spot. It should however be noted that the cases of simultaneous fluctuation reported by Bonfond et al. (2007) were also observed when Io's longitude ranged from $84^{\circ}$ to $99^{\circ}$.

Grodent et al. (2009) reported very similar brightness variations for the Ganymede footprint and suggested two possible origins for them. Either they could be related to magnetic field reconnection at the front of Ganymede's mini magnetosphere, which should re-occur with similar timescales. Another possibility is that they could be related to quasi-periodic apparitions and upward migrations of acceleration structures (i.e. double layers) a few tenths of Jovian radii above the surface. In the case of the IFP, 

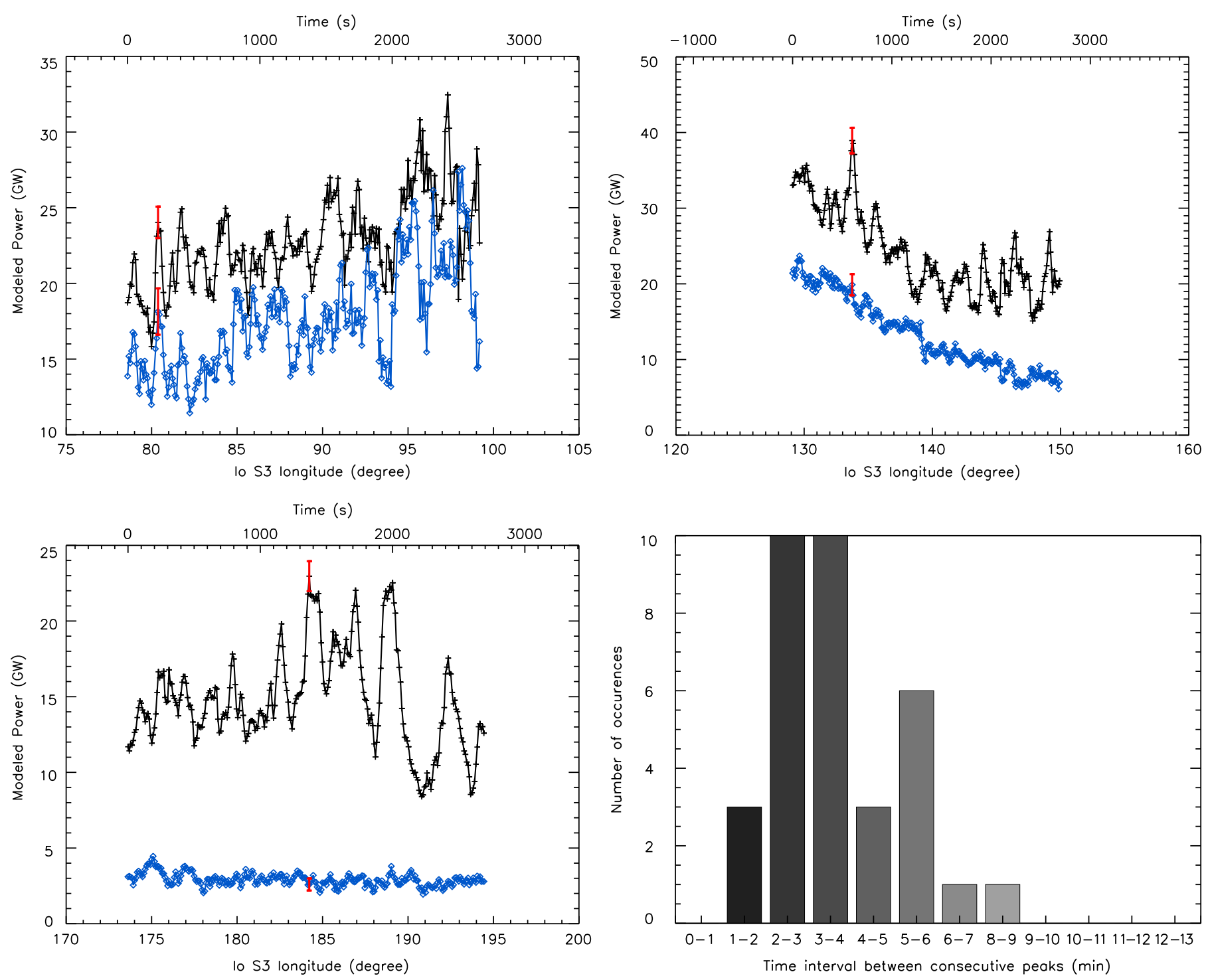

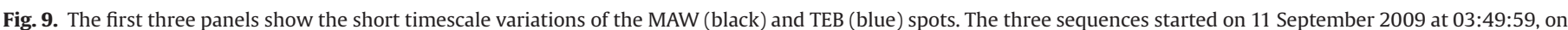

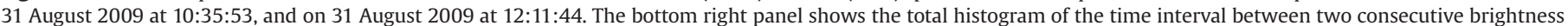

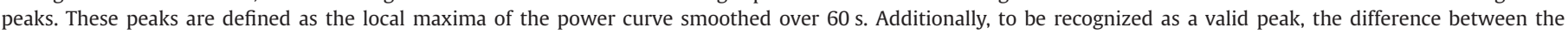

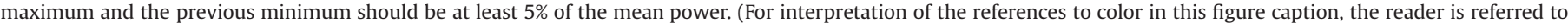
the web version of this article.)

such structures have indeed been identified by Hess et al. (2009) through an analysis of S-burst radio emissions. The mean energy associated with these structures is on the order of $10 \mathrm{eV}$, while the mean energy of the electrons causing the MAW spot is $\sim 1 \mathrm{keV}$ (Bonfond, 2010). The voltage associated with the double layers is thus too small to be the main acceleration process for the precipitating electrons. However, potential jumps as high as $1.5 \mathrm{kV}$ have been reported, which suggests that they could intermittently act as an additional energy source for the electrons and trigger the observed short timescale brightness variations.

In summary, strong variations of the spots' brightness on timescales of 2-4 min are found to be ubiquitous. However, systematic correlations of the MAW and TEB spots' brightness are not confirmed. In the next section, we investigate the brightness changes on timescale of years.

\subsection{Long term brightness variations}

Our dataset gathers 12 years of observations, from 1997 to 2009, and the Io footprint has been observed in similar configurations many times during this period. However, because of the offset of the Jovian magnetic field, and since the Io footprint usually is a target of opportunity only, the Io S3 longitude range where similar configurations have been observed at different times is limited. The torus brightness and density can vary significantly over timescales of months (e.g. Steffl et al., 2004; Nozawa et al., 2004; Bagenal and Delamere, 2011). If the Io footprint brightness was essentially dominated by the torus density, we would expect variations of the spots' brightness from one HST observation campaign to another.

In the northern hemisphere, the Io S3 longitude between 120 and $270^{\circ}$ has been repeatedly observed with STIS and ACS from 1997 to 2007. In this range, Io moves from the center of the torus towards its northern-most location. No significant change in the MAW spot brightness is observed beyond the expected dispersion related to the short-timescale variability (see Fig. 10). The only exception to this rule is a set of 19 images acquired on June 7th 2007, where the Io footprint cannot be distinguished from a patch of emissions usually associated with plasma injections (Bonfond et al., 2012). 


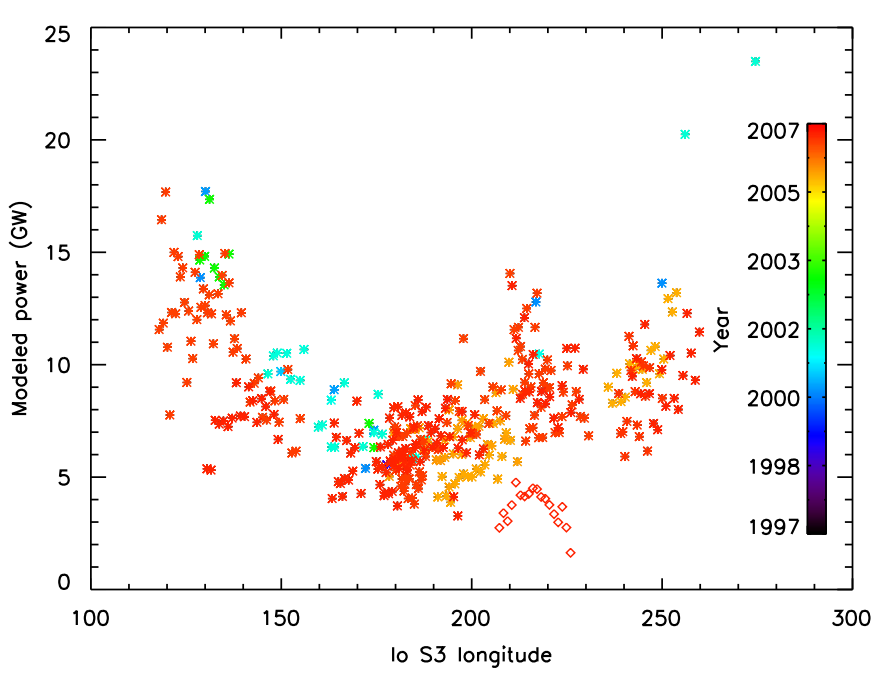

Fig. 10. Plot of the modeled emitted power of the MAW spot in the northern hemisphere. The dates of the observations are color-coded. This plot is dominated by the large 2007 observation campaign concurrent with the New-Horizons fly-by. The spread in the points is real and corresponds to the short-timescale variability of the spots' brightness. However, none of the earlier observations seem to significantly depart from the 2007 trend. The only exception is the arc-shaped set of points around $215^{\circ} \mathrm{S} 3$ Io longitude. These points, shown with diamond symbols, corresponds to a very peculiar case of quasi-disappearance of the IFP discussed in Bonfond et al. (2012). (For interpretation of the references to color in this figure caption, the reader is referred to the web version of this article.)

In the southern hemisphere, the story looks different. Most similar observations took place around $110^{\circ}$, i.e. where Io lies close to the densest part of the torus. Contrary to the northern hemisphere case, very different brightness levels can be seen in these quasi-identical configurations, as testified by the wide spread in the observed and modeled brightness in Figs. 4 and 5. Fig. 11a shows two images acquired 8 years apart as Io's S3 longitude is $\sim 96^{\circ}$, i.e. as Io approaches the torus center from the South. The MAW and TEB spots on the upper image, acquired in 1999, are both brighter and further away from each other than on the lower image. Fig. 11b shows two images acquired $\sim 16$ months apart as Io's S3 longitude is $\sim 155^{\circ}$, i.e. as Io leaves the torus center towards the North. The MAW and TEB spots on the upper image, acquired in 1999, are, this time, fainter and further away from each other than on the lower image. At first sight, it looks like the brightness and the inter-spot distance is not correlated.

Assuming that these changes are related to the changes in the torus density, simulations by Jacobsen (2011) indicate that the torus density has two counteracting effects on the inter-spot distance. On one hand, the inter-spot distance is expected to be directly related to the Alfvén waves travel time and thus to the torus density. The larger the flux tube content, the larger the interspot distance. But on the other hand, if the interaction is strongly non-linear, the intense draping of the magnetic field lines around Io and their increasing alignment with the Alfvén wing would likely decrease the inter-spot distance (see Fig. 12a). Even in the linear case, the increase of the inter-spot distance as a function of the torus density might not be verified as Io crosses the torus center. Because of the one way finite propagation time on the order of $10 \mathrm{~min}$ (Crary and Bagenal, 1997), the MAW and TEB spots overlap a few minutes after Io crosses the torus (see Fig. 12b). The denser the torus, the longer the delay. As a consequence, the possibility remains that, in the case of the 15 August 1999 image, the footprint head looks elongated because it is formed of a TEB spot ahead of a MAW spot. The spots would have overlapped a few minutes before as the torus was relatively tenuous. The 28 December 2000 case would then correspond to a denser torus situation, where the overlap would occur later than in the former

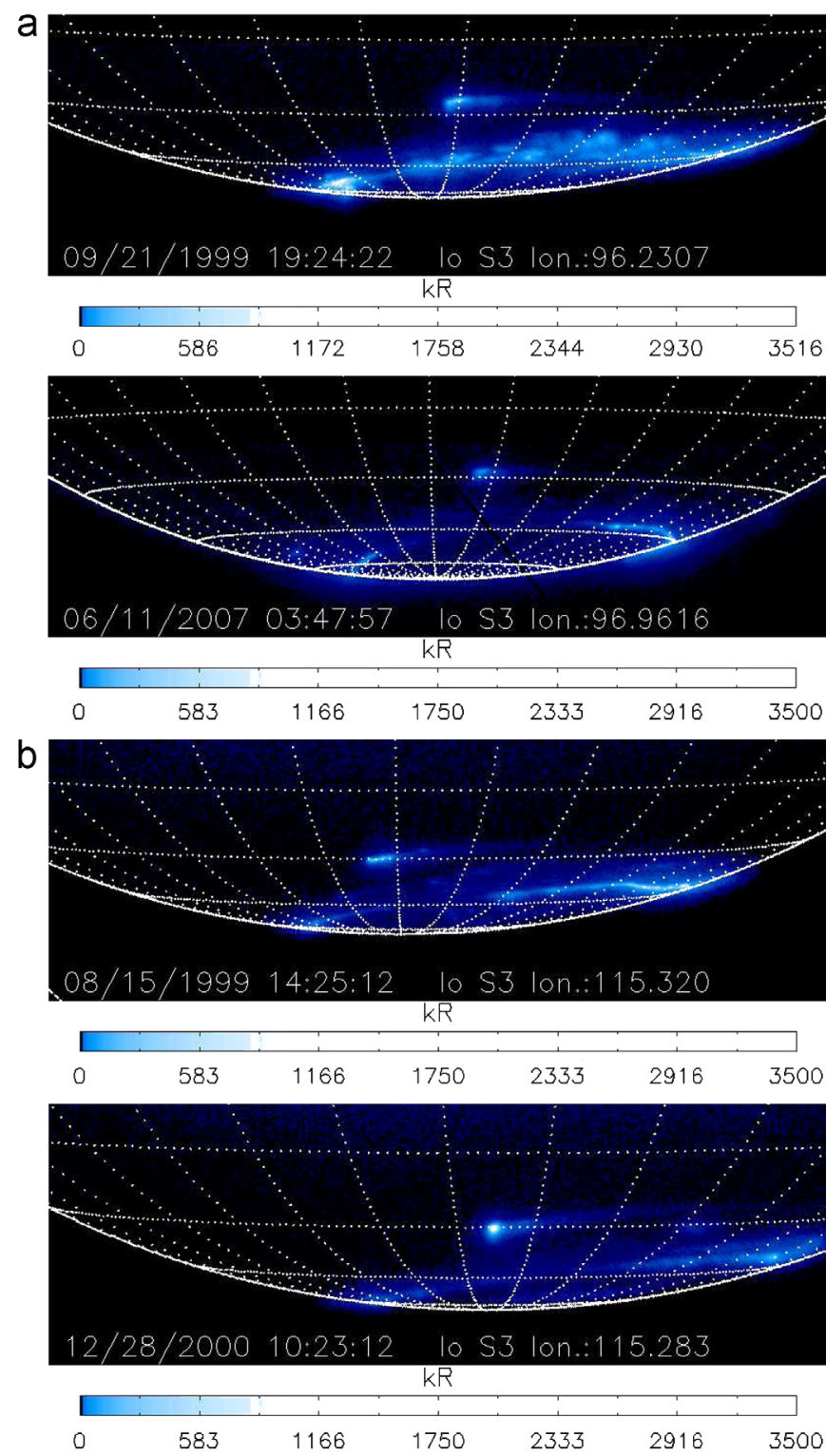

Fig. 11. Panels (a) and (b) show two pairs of images of the southern Io footprint acquired in quasi-identical configurations. The spacing of the grid corresponds to $10^{\circ}$ in planeto-centric latitudes and longitudes. The Io footprint S3 longitude was around $96^{\circ}$ in panel (a) and $115^{\circ}$ in panel (b). It can be seen that even if the location of Io in the torus is the same, the inter-spot distance and the spots' brightness substantially differ.

case and only take place at $115^{\circ}$ Io S3 longitude. It is however not clear whether the large feature seen on 15 August 1999 is due to a TEB spot located upstream or downstream of the MAW spot or even if this picture of juxtaposed spots is too simple as nonlinearities may come into play. Additional and longer observations in this longitude range, allowing to track the location of each spot through time for varying torus conditions, are thus required to sort out this issue. Moreover, the impact of the changes of the torus properties (density, temperature, composition, etc.) on the interspot distance and the spots' overlap timing would require some modeling work beyond the scope of this paper. As a conclusion, it is not clear if the discrepancy between the observed stability of the spots' brightness in the northern hemisphere and the significant variations seen in the southern hemisphere is due to an unfortunate timing, to the characteristics of the hemispheres, or to the different longitude range. 
a

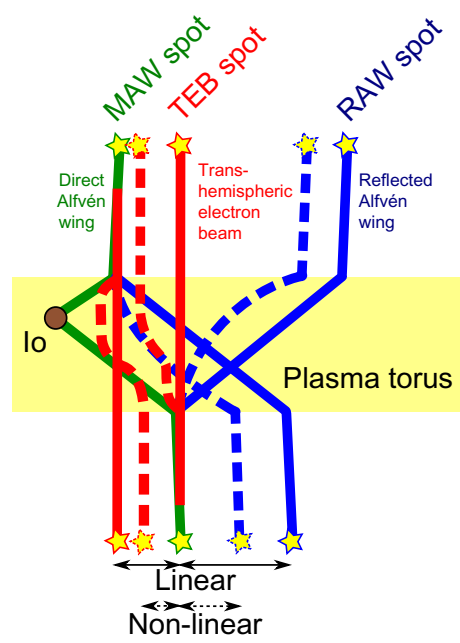

b

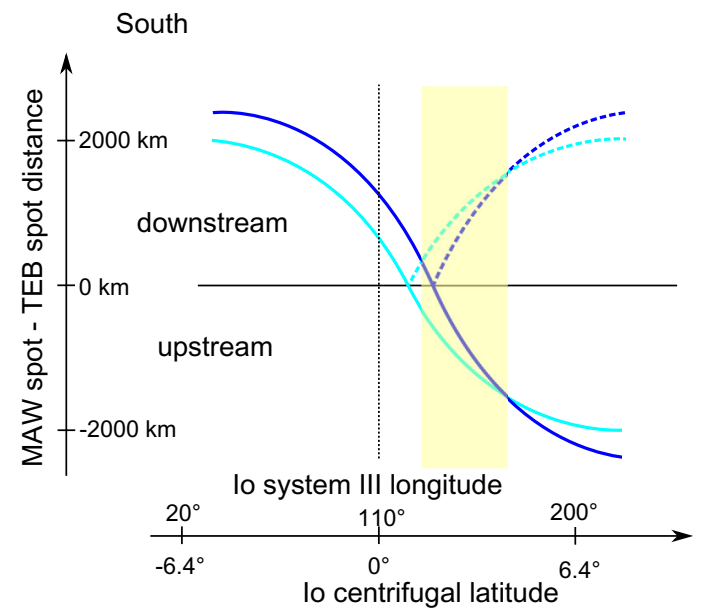

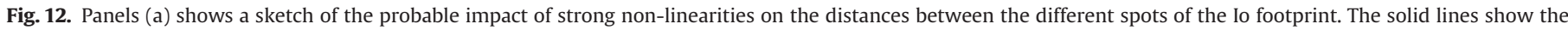

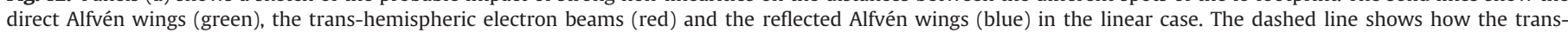

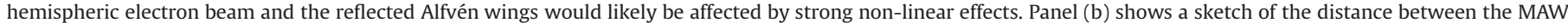

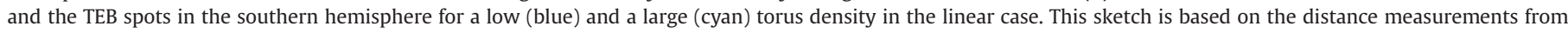

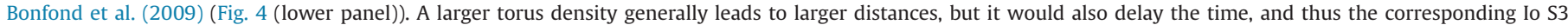

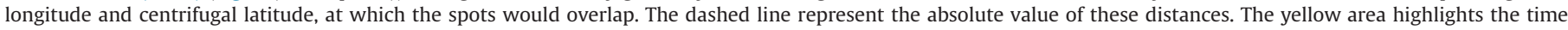

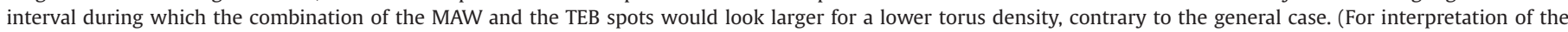
references to color in this figure caption, the reader is referred to the web version of this article.)

The long term variations of the IFP spots' brightness may be summarized as follows: in the northern hemisphere, the measured brightness appears to be stable from year to year. On the contrary, strong brightness and morphological changes over time have been observed in the South.

\section{Conclusions}

The interpretation of the Io footprint brightness requires a good understanding of the observing geometry. For the first time, the brightness estimates reported in the present study are based on measured spatial information such as the absolute and relative spot positions as well as the respective size and altitude of the different features, in lieu of assuming a broad emission region. This procedure consists of assessing how many times simulated spot images with well-known vertical brightness are necessary to best fit the original HST image. When possible, we try to distinguish between the different spots. However, in sectors where the TEB and the MAW merge, i.e. when Io is close to the torus center, they cannot be disentangled. This can partly explain the apparent brightness enhancements of the MAW spot in these locations. Moreover, the strength of the Io-magnetosphere interaction increases in the dense torus center, which is expected to lead to brighter spots in this longitude range as well. It should also be noted that, when the spots are well separated, the brightness ratio between the spots can significantly change. A likely explanation is that the root cause for the correlated brightness variations is common, but the variation of the brightness ratio is a further evidence that each spot is related to a different process.

Another important result is the finding of an asymmetry between the northern and the southern spots' brightness. Moreover, the southern spots' brightness at $110^{\circ}$ is much larger than at $290^{\circ}$ while Io is at the same centrifugal latitude in the torus. This information indicates that the centrifugal latitude of Io is not the only parameter controlling the spots' brightness, but that other processes such as the modulations of the power transmission along the Alfvén wing, of the power transfer to the precipitating electron or the size of the loss cone also play a major role. The relative contribution of these different processes is explored in Paper II.

We also confirmed the presence of strong brightness variations on timescale of 2-4 min for both the MAW and the TEB spots. The fluctuation of the two spots are generally not correlated, except possibly in the $78^{\circ}-94^{\circ}$ System III sector. These fast brightness variations appear similar to the fastest brightness variations of the Ganymede footprint reported by Grodent et al. (2009). A previous analysis of Io related S-burst radio emissions revealed the existence of vertically drifting acceleration structures located at approximately $0.1-0.3$ Jovian radii above the Jovian surface (i.e. 1 bar level) (see Fig. 1) (Hess et al., 2009). These variable acceleration structures had a reappearance quasi-period of $\sim 3 \mathrm{~min}$. They are not energetic enough to be the main cause for the electron acceleration. However the similarity of the observed timescales suggests that they could possibly provide some additional energy to the precipitating electrons and trigger the fast brightness fluctuations.

Finally, the maximum vertical brightness that we derive implies precipitating electron energy fluxes between 250 and $2000 \mathrm{~mW} / \mathrm{m}^{2}$ for the MAW spot, while previous estimates were lying closer to 2.3-23 mW/ $\mathrm{m}^{2}$ (Gérard et al., 2006). For a series of reasons detailed above, our estimates of the total precipitated power is larger than the values inferred in some previous ones. These large values indicate that the energy transfer from the initial interaction at Io to the acceleration of electrons into the Jovian atmosphere is a very efficient process, which requires a high level of filamentation of the Alfvén waves before they escape the torus (Hess et al., 2010). Moreover, such an intense and localized energy input is likely to trigger a strong atmospheric response, such as vertical winds or an enhanced vertical mixing. Both mechanisms would possibly bring hydrocarbons above the usual homopause level and thus increase the absorption of UV emissions. The modeling of this sudden heating in the Jovian upper atmosphere could possibly explain the discrepancy between the estimates of the energy of the electrons creating the IFP based on color ratios (Gérard et al., 2002) and those based on the altitude profile (Bonfond, 2010). 


\section{Acknowledgments}

B.B. J.-C.G., D.G. were supported by the PRODEX program managed by ESA in collaboration with the Belgian Federal Science Policy Office. A.R. was funded by the Belgian Fund for Scientific Research (F.R.S.-FNRS). J.C. was supported by NASA grants HST-6011649.01-A and HST-60-10862.01-A from STScI to Boston University. This research is based on observations made with the Hubble Space Telescope obtained at the Space Telescope Science Institute, which is operated by AURA Inc.

\section{References}

Bagenal, F., Delamere, P.A., 2011. Flow of mass and energy in the magnetospheres of Jupiter and Saturn. Journal of Geophysical Research (Space Physics) 116, 5209. Baker, D.J., 1974. Rayleigh, the unit for light radiance. Applied Optics 13, 2160-2163. Bonfond, B., 2009. Morphology and Dynamics of the Io UV Footprint. Ph.D. Thesis, Université de Liège urn:ULgetd-02102010-140716.

Bonfond, B., 2010. The 3-D extent of the Io UV footprint on Jupiter. Journal of Geophysical Research (Space Physics) 115, A09217.

Bonfond, B., Gérard, J.C., Grodent, D., Saur, J., 2007. Ultraviolet Io footprint short timescale dynamics. Geophysical Research Letters 34, L06201.

Bonfond, B., Grodent, D., Gérard, J.C., Radioti, A., Dols, V., Delamere, P.A., Clarke, J.T., 2009. The Io UV footprint: location, inter-spot distances and tail vertical extent. Journal of Geophysical Research 114, A07224.

Bonfond, B., Grodent, D., Gérard, J.C., Radioti, A., Saur, J., Jacobsen, S., 2008. UV Io footprint leading spot: a key feature for understanding the UV Io footprint multiplicity?. Geophysical Research Letters 35, L05107.

Bonfond, B., Grodent, D., Gérard, J.C., Stallard, T., Clarke, J.T., Yoneda, M., Radioti, A., Gustin, J., 2012. Auroral evidence of Io's control over the magnetosphere of Jupiter. Geophysical Research Letters 39, 1105.

Bonfond, B., Vogt, M.F., Gérard, J.C., Grodent, D., Radioti, A., Coumans, V., 2011. Quasi-periodic polar flares at Jupiter: a signature of pulsed dayside reconnections?. Geophysical Research Letters 380, L02104.

Chust, T., Roux, A., Kurth, W.S., Gurnett, D.A., Kivelson, M.G., Khurana, K.K., 2005. Are Io's Alfvén wings filamented? Galileo observations. Planetary and Space Science 53, 395-412.

Clarke, J.T., Ajello, J., Ballester, G., Ben Jaffel, L., Connerney, J., Gérard, J.C., Gladstone G.R. Grodent, D. Pryor, W. Trauger, J. Waite, J.H., 2002. Ultraviolet emissions from the magnetic footprints of Io, Ganymede and Europa on Jupiter. Nature 415, 997-1000.

Clarke, J.T., Ballester, G.E., Trauger, J., Ajello, J., Pryor, W., Tobiska, K., Connerney, J.E. P., Gladstone, G.R., Waite, J.H., Ben Jaffel, L., Gérard, J.C., 1998. Hubble space telescope imaging of Jupiter's UV aurora during the Galileo orbiter mission. Journal of Geophysical Research 103, 20217-20236.

Clarke, J.T., Ballester, G.E., Trauger, J., Evans, R., Connerney, J.E.P., Stapelfeldt, K. Crisp, D., Feldman, P.D., Burrows, C.J., Casertano, S., Gallagher III, J.S., Griffiths, R. E., Hester, J.J., Hoessel, J.G., Holtzman, J.A., Krist, J.E., Meadows, V., Mould, J.R., Scowen, P.A., Watson, A.M., Westphal, J.A., 1996. Far-ultraviolet imaging of Jupiter's Aurora and the lo footprint. Science 274, 404-409.

Connerney, J.E.P., Baron, R., Satoh, T., Owen, T., 1993. Images of excited $H_{3}^{+}$at the foot of the Io flux tube in Jupiter's atmosphere. Science 262, 1035-1038.

Crary, F.J., Bagenal, F., 1997. Coupling the plasma interaction at Io to Jupiter. Geophysical Research Letters 24, pp. 2135-2138.

Delamere, P.A., Bagenal, F., Ergun, R., Su, Y.J., 2003. Momentum transfer between the Io plasma wake and Jupiter's ionosphere. Journal of Geophysical Research 108, 1-11.

Gérard, J.C., Gustin, J., Grodent, D., Delamere, P., Clarke, J.T., 2002. Excitation of the FUV Io tail on Jupiter: characterization of the electron precipitation. Journal of Geophysical Research 107, 1-30.

Gérard, J.C., Saglam, A., Grodent, D., Clarke, J.T., 2006. Morphology of the ultraviolet Io footprint emission and its control by Io's location. Journal of Geophysical Research 111, A04202.

Gérard, J.C., Singh, V., 1982. A model of energy deposition of energetic electrons and EUV emission in the Jovian and Saturnian atmospheres and implications. Journal of Geophysical Research 87, 4525-4532.

Gledhill, J.A., 1967. Magnetosphere of Jupiter. Nature 214, 155-156.

Grodent, D., Bonfond, B., Radioti, A., Gérard, J.C., Jia, X., Nichols, J.D., Clarke, J.T. 2009. Auroral footprint of Ganymede. Journal of Geophysical Research 114, A07212.

Grodent, D., Gladstone, G.R., Gerard, J.C., Dols, V., Waite, J.H., 1997. Simulation of the morphology of the Jovian UV North Aurora Observed with the Hubble Space Telescope. Icarus 128, 306-321.
Gurnett, D.A., Goertz, C.K., 1981. Multiple Alfven wave reflections excited by Io Origin of the Jovian decametric arcs. Journal of Geophysical Research 86, $717-722$.

Gustin, J., Bonfond, B., Grodent, D., Gérard, J.C., 2012. Conversion from hst acs and stis auroral counts into brightness, precipitated power and radiated power for h2 giant planets. Journal of Geophysical Research 117, 7316.

Hess, S., Bonfond, B., Chantry, V., Gérard, J.C., Grodent, D., Jacobsen, S., Radioti, A. Evolution of the Io footprint brightness II: modeling. Planetary Space Science, this issue.

Hess, S., Zarka, P., Mottez, F., Ryabov, V.B., 2009. Electric potential jumps in the IoJupiter flux tube. Planetary Space and Science 57, 23-33.

Hess, S.L.G., Delamere, P., Dols, V., Bonfond, B., Swift, D., 2010. Power transmission and particle acceleration along the Io flux tube. Journal of Geophysical Research 115, A06205.

Jacobsen, S., 2011. Three-Dimensional Magnetohydrodynamic Simulations of Io's Non-Linear Interaction with the Jovian Magnetosphere. Ph.D. Thesis, Universität zu Köln.

Jacobsen, S., Saur, J., Neubauer, F.M., Bonfond, B., Gérard, J.C., Grodent, D., 2010. Location and spatial shape of electron beams in Io's wake. Journal of Geophysical Research 115, A04205.

Jones, S.T., Su, Y.J., 2008. Role of dispersive Alfvén waves in generating parallel electric fields along the Io-Jupiter fluxtube. Journal of Geophysical Research 113, A12205.

Kivelson, M.G., Bagenal, F., Kurth, W.S., Neubauer, F.M., Paranicas, C., Saur, J., 2004. Magnetospheric interactions with satellites. Jupiter The Planet, Satellites and Magnetosphere, pp. 513-536.

Krist, J., Hook, R., 2004. The Tiny Tim User's Guide Version 6.3. Space Telescope Science Institute.

Magain, P., Courbin, F., Gillon, M., Sohy, S., Letawe, G., Chantry, V., Letawe, Y., 2007. A deconvolution-based algorithm for crowded field photometry with unknown point spread function. Astronomy and Astrophysics 461, 373-379 arXiv: astro-ph/0609600.

Neubauer, F.M., 1980. Nonlinear standing Alfven wave current system at IoTheory. Journal of Geophysical Research 85, 1171-1178.

Nozawa, H., Misawa, H., Takahashi, S., Morioka, A., Okano, S., Sood, R., 2004. Longterm variability of [SII] emissions from the Io plasma torus between 1997 and 2000. Journal of Geophysical Research (Space Physics) 109, 7209.

Pavlovsky, C., et al., 2006. Advanced Camera for Surveys Instrument Handbook for Cycle 16. STScI. version 7.1 edition. 〈Http://www.stsci.edu/hst/acs〉.

Prangé, R., Rego, D., Pallier, L., Connerney, J., Zarka, P., Queinnec, J., 1998. Detailed study of FUV Jovian auroral features with the post-COSTAR HST faint object camera. Journal of Geophysical Research 103, 20195-20216.

Prangé, R., Rego, D., Southwood, D., Zarka, P., Miller, S., Ip, W., 1996. Rapid energy dissipation and variability of the Io-Jupiter electrodynamic circuit. Nature 379, 323-325.

Saur, J., Grambusch, T., Duling, S., Neubauer, F.M., Simon, S., 2013. Magnetic energy fluxes in sub-Alfvénic planet star and moon planet interactions. Astronomy and Astrophysics 552, A119.

Saur, J., Neubauer, F.M., Connerney, J.E.P., Zarka, P., Kivelson, M.G., 2004. Plasma interaction of Io with its plasma torus. Jupiter. The Planet, Satellites and Magnetosphere. Cambridge University Press, pp. 537-560.

Saur, J., Neubauer, F.M., Strobel, D.F., Summers, M.E., 1999. Three-dimensional plasma simulation of Io's interaction with the Io plasma torus: asymmetric plasma flow. Journal of Geophysical Research 104, 25105-25126.

Serio, A.W., Clarke, J.T., 2008. The variation of Io's auroral footprint brightness with the location of Io in the plasma torus. Icarus 197, 368-374.

Shannon, C.E., 1949. Communications in the presence of noise. In: Proceedings of the IRE.

Steffl, A.J., Stewart, A.I.F., Bagenal, F., 2004. Cassini UVIS observations of the Io plasma torus. I. Initial results. Icarus 172, 78-90.

Swift, D.W., 2007. Simulation of auroral electron acceleration by inertial Alfvén waves. Journal of Geophysical Research 112, A12207.

Wannawichian, S., Clarke, J.T., Nichols, J.D., 2010. Ten years of Hubble Space Telescope observations of the variation of the Jovian satellites' auroral footprint brightness. Journal of Geophysical Research (Space Physics) 115, A02206.

Wannawichian, S.T., Bagenal, C.J., Smyth, F., Paterson, C.A., Nichols, J.D. Longitudinal modulation of the brightness of io's auroral footprint emission: comparison with models. Journal of Geophysical Research, http://dx.doi.org/10.1002/ jgra.50346, in press.

Wilkinson, M.H., 1998. Evidence for periodic modulation of Jupiter's decametric radio emission. Journal of Geophysical Research 1031, 19985-19992.

Williams, D.J., Thorne, R.M., 2003. Energetic particles over Io's polar caps. Journal of Geophysical Research 108, 1-7.

Wright, A.N., 1987. The interaction of Io's Alfven waves with the Jovian magnetosphere. Journal of Geophysical Research 92, 9963-9970. 